\title{
On instabilities of scalar hairy regular compact reflecting stars
}

\section{Yan Peng}

School of Mathematical Sciences, Qufu Normal University, No.57 Jingxuan West Road, Qufu Shandong 273165, China

E-mail: yanpengphy@163.com

\begin{abstract}
We study the system constructed by charged scalar fields linearly coupled to asymptotically flat horizonless compact reflecting stars. We obtain bounds on the charge of the scalar field, below which the scalar hairy star is expected to suffer from nonlinear instabilities. It means that scalar hairy regular configurations are unstable for scalar fields of small charge. For the highly-charged star, there are also bounds on radii of regular compact reflecting stars. When the star radius is below the bound, the hairy star is always unstable.
\end{abstract}

KEYwords: Black Holes, Classical Theories of Gravity

ARXiv EPRINT: 1810.04102 


\section{Contents}

1 Introduction 1

2 The scalar field and reflecting star gravity system 2

3 Bounds for the charge of scalar fields supported by reflecting stars $\quad 3$

3.1 Bounds of the scalar field charge in the case of $\frac{Q}{M} \leqslant 1 \quad 3$

3.2 Bounds of the scalar field charge in the case of $1<\frac{Q}{M} \leqslant \sqrt{\frac{9}{8}} \quad 5$

4 Conclusions $\quad 5$

\section{Introduction}

A well known characteristic of classical black holes is the no scalar hair theorem [1-5], which states that static scalar fields cannot condense outside black holes in the asymptotically flat background, see references [6]-[11] and reviews [12, 13]. And it was usually believed that this no hair property is due to the existence of black hole horizons, which can absorb matter fields and radiations. So it is interesting to further examine whether this no hair behavior is restricted to the spacetime with a horizon.

Hod firstly proved that the neutral static massive scalar field cannot condense outside the horizonless compact reflecting stars in the asymptotically flat gravity [14]. And the asymptotically flat regular reflecting star also cannot support the neutral massless scalar field nonminimally coupled to the gravity [15]. Including a positive cosmological constant, it was shown that there is also no hair theorem for the neutral scalar field in the asymptotically dS regular reflecting star spacetime [16]. It means that the neutral scalar hair usually cannot form outside the reflecting star but the system composed of charged scalar fields and charged horizonless backgrounds is still to be studied. In fact, the regular configurations with charged scalar hair supported by a charged reflecting shell were constructed when the shell radius is below an upper bound and above the upper bound, the scalar field cannot exist outside the shell [17-19]. And in the reflecting star background, an upper bound for the radius of the charged star with charged hair was also obtained and the no scalar hair theorem still holds in the case that the star radius is above the upper bound [20-27].

On the other side, the general relativity predicts that closed light rings may exist outside compact objects, such as black holes and horizonless compact stars [28-32]. It was stated that horizonless compact stars with stable closed light rings are expected to develop nonlinear instabilities due to the fact that massless perturbation fields tend to pile up on stable null geodesics [33-35]. And it was shown that the innermost null circular geodesic of a horizonless compact object is stable [36, 37]. So regular compact objects with outermost light rings above the object surface are dynamically unstable [38]. We should 
emphasize that the dynamical instability is expected to develop for compact objects with stable null circular geodesics which are located inside the object. At present, various types of regular scalar hairy configurations supported by compact reflecting stars have been constructed, but to the best of knowledge, (in)stabilities of these scalar hairy stars hasn't been studied. In this work, we plan to disclose the (in)stability of the hairy reflecting star through behaviors of light rings.

The rest of this work is planed as follows. In section 2, we introduce the system constructed by a charged scalar field linearly coupled to a charged reflecting star. And in section 3, we obtain bounds on the star radius and scalar field charge, which can be used to describe the instability of the hairy star. We will summarize main results at the last section.

\section{The scalar field and reflecting star gravity system}

We are interested in the system constructed by a charged scalar field coupled to the charged compact reflecting star in the asymptotically flat background. And the matter field Lagrange density reads

$$
\mathcal{L}=-\frac{1}{4} F^{M N} F_{M N}-\left|\nabla_{\mu} \psi-q A_{\mu} \psi\right|^{2}-m^{2} \psi^{2} .
$$

Here $\psi(r)$ and $A_{\mu}$ are the scalar field and Maxwell field respectively. We also label q as the scalar field charge and $\mathrm{m}$ as the scalar field mass.

The general spherically symmetric compact star solution can be expressed as [28]

$$
d s^{2}=-e^{-2 \chi} f d t^{2}+\frac{d r^{2}}{f}+r^{2}\left(d \theta^{2}+\sin ^{2} \theta d \varphi^{2}\right)
$$

where $\chi(r)$ and $f(r)$ are metric functions satisfying $\chi(r \rightarrow \infty) \rightarrow 0$ and $f(r \rightarrow \infty) \rightarrow 1$. We define $r_{s}$ as the star radius and there is $f(r)>0$ for $r \geqslant r_{s}$ since we study the regular compact star.

According to the Einstein equations $G_{\nu}^{\mu}=8 \pi T_{\nu}^{\mu}$, the metric equations are [38-42]

$$
\begin{aligned}
& f^{\prime}=-8 \pi r\left[\rho+\frac{Q^{2}(r)}{8 \pi r^{4}}\right]+(1-f) / r \\
& \chi^{\prime}=-4 \pi r(\rho+p) / f
\end{aligned}
$$

with $T_{t}^{t}=-\rho, T_{r}^{r}=p$ and $Q(r)$ is the electric charge contained within a sphere of area of radius $\mathrm{r}$.

In the present paper, we are interested in neglecting the scalar hair's backreaction on the metric and there is $\chi(r)=0$ and $f(r)=1-\frac{2 M}{r}+\frac{Q^{2}}{r^{2}}$ with $M$ as the ADM mass and $Q$ corresponding to the star charge. We also assume that the Maxwell field has only the nonzero $t$ component in the form $A_{t}=-\frac{Q}{r} d t$. And the equation of radial dependence scalar field $\psi=\psi(r)$ is

$$
\psi^{\prime \prime}+\left(\frac{2}{r}+\frac{f^{\prime}}{f}\right) \psi^{\prime}+\left(\frac{q^{2} Q^{2}}{r^{2} f^{2}}-\frac{m^{2}}{f}\right) \psi=0
$$

with $f=1-\frac{2 M}{r}+\frac{Q^{2}}{r^{2}}[24,43-47]$. 
The compact star has a scalar reflecting surface that the scalar field vanishes at the star radius $r_{s}$. At the infinity, the scalar field possesses the asymptotical behavior of $\psi \sim A \cdot \frac{1}{r} e^{-m r}+B \cdot \frac{1}{r} e^{m r}$ with $\mathrm{A}$ and $\mathrm{B}$ as integral constants. We set $B=0$ to get the physical scalar field solution and boundary conditions of the scalar field are

$$
\psi\left(r_{s}\right)=0, \quad \psi(\infty)=0
$$

According to the general relativity, closed light rings may exist outside compact stars. In this work, the outer light ring of the charged hairy compact star is $r_{\gamma}=\frac{1}{2}(3 M+$ $\left.\sqrt{9 M^{2}-8 Q^{2}}\right)$, which refers to the outer null circular geodesic above the surface of the compact object where the contribution of the linearized field to the spacetime metric is neglected [38]. In fact, the innermost circular light ring of a horizonless compact object is stable. And it was stated that horizonless compact stars are unstable if the stars possess stable closed light rings [33-35]. We realize that the former studies have shown that generic horizonless compact objects are characterized by an even number of light rings. Thus, the presence of a light ring outside the objects implies the presence of an inner light ring as well [36]. So we can disclose the stability of hairy stars by examining whether there is the outer light ring outside the star surface.

\section{Bounds for the charge of scalar fields supported by reflecting stars}

\subsection{Bounds of the scalar field charge in the case of $\frac{Q}{M} \leqslant 1$}

Introducing a new radial function $\tilde{\psi}=\sqrt{r} \psi$, the scalar field equation (2.5) is transformed into

$$
r^{2} \tilde{\psi}^{\prime \prime}+\left(r+\frac{r^{2} f^{\prime}}{f}\right) \tilde{\psi}^{\prime}+\left(-\frac{1}{4}-\frac{r f^{\prime}}{2 f}+\frac{q^{2} Q^{2}}{f^{2}}-\frac{m^{2} r^{2}}{f}\right) \tilde{\psi}=0
$$

where $f=1-\frac{2 M}{r}+\frac{Q^{2}}{r^{2}}$.

From the relation (2.6), we get the following boundary conditions

$$
\tilde{\psi}\left(r_{s}\right)=0, \quad \tilde{\psi}(\infty)=0 .
$$

One extremum point $r=r_{\text {peak }}$ of the function $\tilde{\psi}$ exists between the star surface $r_{s}$ and the infinity boundary. At this extremum point $r_{\text {peak }}$, the following relation holds

$$
\left\{\tilde{\psi}^{\prime}=0 \quad \text { and } \quad \tilde{\psi} \tilde{\psi}^{\prime \prime} \leqslant 0\right\} \quad \text { for } \quad r=r_{\text {peak }} .
$$

With the relations (3.1) and (3.3), we obtain the inequality

$$
-\frac{1}{4}-\frac{r f^{\prime}}{2 f}+\frac{q^{2} Q^{2}}{f^{2}}-\frac{m^{2} r^{2}}{f} \geqslant 0 \quad \text { for } \quad r=r_{\text {peak }} .
$$

It can be transformed into

$$
m^{2} r^{2} f(r) \leqslant q^{2} Q^{2}-\frac{r f f^{\prime}}{2}-\frac{1}{4} f^{2} \quad \text { for } \quad r=r_{\text {peak }} .
$$


Firstly, we have $r_{s} \geqslant M+\sqrt{M^{2}-Q^{2}}$ since there is an horizon above the surface of the compact object in the case of $r_{s}<M+\sqrt{M^{2}-Q^{2}}$. Considering $r_{s} \geqslant M+\sqrt{M^{2}-Q^{2}}$, the following relations hold

$$
\begin{aligned}
r \geqslant r_{s} \geqslant M+\sqrt{M^{2}-Q^{2}} \geqslant M & \geqslant Q, \\
f=1-\frac{2 M}{r}+\frac{Q^{2}}{r^{2}}=\frac{1}{r^{2}}\left[(r-M)^{2}-\left(M^{2}-Q^{2}\right)\right] & \geqslant 0, \\
r f^{\prime}=r\left(\frac{2 M}{r^{2}}-\frac{2 Q^{2}}{r^{3}}\right)=\frac{2 M}{r}\left(1-\frac{Q}{r} \frac{Q}{M}\right) & \geqslant 0, \\
\left(r^{2} f\right)^{\prime}=\left(r^{2}-2 M r+Q^{2}\right)^{\prime}=2(r-M) & \geqslant 0 .
\end{aligned}
$$

From relations (3.5) and (3.9), $r^{2} f$ is an increasing function and we have

$$
m^{2} r_{s}^{2} f\left(r_{s}\right) \leqslant m^{2} r^{2} f(r) \leqslant q^{2} Q^{2}-\frac{r f f^{\prime}}{2}-\frac{1}{4} f^{2} \leqslant q^{2} Q^{2} \quad \text { for } \quad r=r_{\text {peak }}
$$

According to (3.10), there is $m^{2} r_{s}^{2} f\left(r_{s}\right) \leqslant q^{2} Q^{2}$ or

$$
m^{2} r_{s}^{2}\left(1-\frac{2 M}{r_{s}}+\frac{Q^{2}}{r_{s}^{2}}\right) \leqslant q^{2} Q^{2}
$$

The inequality can also be transformed into

$$
\left(m r_{s}\right)^{2}-(2 m M)\left(m r_{s}\right)+Q^{2}\left(m^{2}-q^{2}\right) \leqslant 0 .
$$

With the relation (3.12), we obtain bounds on radii of hairy stars as

$$
m r_{s} \leqslant m M+\sqrt{m^{2}\left(M^{2}-Q^{2}\right)+q^{2} Q^{2}}
$$

with dimensionless quantities according to the symmetry

$$
r \rightarrow k r, \quad m \rightarrow m / k, \quad M \rightarrow k M, \quad Q \rightarrow k Q, \quad q \rightarrow q / k .
$$

In order to obtain the instability condition, we impose that the outer light ring is above the upper bound (3.13) in the form

$$
m r_{\gamma}=\frac{1}{2}\left(3 m M+\sqrt{9 m^{2} M^{2}-8 m^{2} Q^{2}}\right) \geqslant m M+\sqrt{m^{2}\left(M^{2}-Q^{2}\right)+q^{2} Q^{2}} .
$$

From (3.15), we get the upper bound for the charge of the scalar field as

$$
\left(\frac{q}{m}\right)^{2} \leqslant \frac{3 M^{2}}{2 Q^{2}}+\frac{M \sqrt{9 M^{2}-8 Q^{2}}}{2 Q^{2}}-1
$$

So for $\frac{Q}{M} \leqslant 1$ in this part, we find that the hairy star is unstable for small scalar field charge below the bound (3.16). 


\subsection{Bounds of the scalar field charge in the case of $1<\frac{Q}{M} \leqslant \sqrt{\frac{9}{8}}$}

Now we extend the discussion of (in)stabilities of hairy reflecting stars to the range of $1<\frac{Q}{M} \leqslant \sqrt{\frac{9}{8}}$. In the case of $r_{s}<\frac{Q^{2}}{M}$, there is

$$
r_{\gamma}=\frac{1}{2}\left(3 M+\sqrt{9 M^{2}-8 Q^{2}}\right) \geqslant \frac{3 M}{2} \geqslant \frac{3}{2} \sqrt{\frac{8}{9}} Q=\sqrt{2} Q \geqslant \sqrt{\frac{9}{8}} Q \geqslant \frac{Q}{M} Q>r_{s} .
$$

So the light ring $r_{\gamma}$ is above the star radius $r_{s}$ and the star is unstable.

In another case of $r_{s} \geqslant \frac{Q^{2}}{M}$, we have

$$
\begin{array}{r}
Q^{2}-M^{2} \geqslant 0, \\
1-\frac{Q}{r} \frac{Q}{M} \geqslant 1, \\
r \geqslant r_{s} \geqslant \frac{Q^{2}}{M} \geqslant Q \geqslant M .
\end{array}
$$

And the following relations exist

$$
\begin{array}{r}
f=1-\frac{2 M}{r}+\frac{Q^{2}}{r^{2}}=\frac{1}{r^{2}}\left[(r-M)^{2}+\left(Q^{2}-M^{2}\right)\right] \geqslant 0, \\
r f^{\prime}=r\left(\frac{2 M}{r^{2}}-\frac{2 Q^{2}}{r^{3}}\right)=\frac{2 M}{r}\left(1-\frac{Q}{r} \frac{Q}{M}\right) \geqslant 0, \\
\left(r^{2} f\right)^{\prime}=\left(r^{2}-2 M r+Q^{2}\right)^{\prime}=2(r-M) \geqslant 0 .
\end{array}
$$

Following approaches in part A, we again obtain the upper bound for hairy star radius

$$
m r_{s} \leqslant m M+\sqrt{m^{2}\left(M^{2}-Q^{2}\right)+q^{2} Q^{2}} .
$$

The same upper bound was obtained in [24] on conditions that $\frac{Q}{M} \leqslant 1$, which was also shown in (3.13) of subsection 3.1. Here we find that the same bound holds in the other case of $1<\frac{Q}{M} \leqslant \sqrt{\frac{9}{8}}$.

And we also arrive at the bound on the scalar field charge the same as (3.16) in the form

$$
\left(\frac{q}{m}\right)^{2} \leqslant \frac{3 M^{2}}{2 Q^{2}}+\frac{M \sqrt{9 M^{2}-8 Q^{2}}}{2 Q^{2}}-1
$$

It is well know that the neutral scalar field usually cannot exist around reflecting stars and charge scalar fields may condense outside the reflecting star. In this part with $1<\frac{Q}{M} \leqslant \sqrt{\frac{9}{8}}$, we show that the hairy star is always unstable on the condition $r_{s}<\frac{Q^{2}}{M}$ and when $r_{s} \geqslant \frac{Q^{2}}{M}$, the hairy star is still unstable for small charge of scalar fields below the bound (3.25).

\section{Conclusions}

We studied the system of charged scalar fields linearly coupled to regular reflecting stars. In a parameter range of $\frac{Q}{M} \leqslant \sqrt{\frac{9}{8}}$, we obtained upper bounds for hairy star radii as 
$m r_{s} \leqslant m M+\sqrt{m^{2}\left(M^{2}-Q^{2}\right)+q^{2} Q^{2}}$, where $m$ and $q$ are the mass and charge of the scalar field respectively, $\mathrm{M}$ serves as the ADM mass and Q corresponds to the star charge. Scalar fields can condense only when the star radius is below the upper bound. And we mainly further investigated (in)stabilities of hairy reflecting stars. We divided the discussion into two cases as follows.

(1) In the first case of $\frac{Q}{M} \leqslant 1$, the hairy star is expected to suffer from nonlinear instabilities when the charge of the scalar field is small expressed with dimensionless quantities as $\left(\frac{q}{m}\right)^{2} \leqslant \frac{3 M^{2}}{2 Q^{2}}+\frac{M \sqrt{9 M^{2}-8 Q^{2}}}{2 Q^{2}}-1$.

(2) In the second case of $1<\frac{Q}{M} \leqslant \sqrt{\frac{9}{8}}$, we found an upper bound for the hairy star radius as $r_{s}<\frac{Q^{2}}{M}$, below which the hairy star is unstable and when the star radius is above this bound, the hairy star is still unstable in the case that the charge of the scalar field satisfies $\left(\frac{q}{m}\right)^{2} \leqslant \frac{3 M^{2}}{2 Q^{2}}+\frac{M \sqrt{9 M^{2}-8 Q^{2}}}{2 Q^{2}}-1$.

\section{Acknowledgments}

We would like to thank the anonymous referee for the constructive suggestions to improve the manuscript. This work was supported by the Shandong Provincial Natural Science Foundation of China under Grant No. ZR2018QA008.

Open Access. This article is distributed under the terms of the Creative Commons Attribution License (CC-BY 4.0), which permits any use, distribution and reproduction in any medium, provided the original author(s) and source are credited.

\section{References}

[1] J.D. Bekenstein, Transcendence of the law of baryon-number conservation in black hole physics, Phys. Rev. Lett. 28 (1972) 452 [INSPIRE].

[2] J.E. Chase, Event horizons in static scalar-vacuum space-times, Commun. Math. Phys. 19 (1970) 276.

[3] C. Teitelboim, Nonmeasurability of the baryon number of a black-hole, Lett. Nuovo Cim. 3S2 (1972) 326 [INSPIRE].

[4] J.D. Bekenstein, Black-hole thermodynamics, Phys. Today 33 (1980) 24.

[5] R. Ruffini and J.A. Wheeler, Introducing the black hole, Phys. Today 24 (1971) 30.

[6] S. Hod, Stationary Scalar Clouds Around Rotating Black Holes, Phys. Rev. D 86 (2012) 104026 [Erratum ibid. D 86 (2012) 129902] [arXiv:1211.3202] [INSPIRE].

[7] C.A.R. Herdeiro and E. Radu, Kerr black holes with scalar hair, Phys. Rev. Lett. 112 (2014) 221101 [arXiv: 1403.2757] [INSPIRE].

[8] C.L. Benone, L.C.B. Crispino, C. Herdeiro and E. Radu, Kerr-Newman scalar clouds, Phys. Rev. D 90 (2014) 104024 [arXiv: 1409.1593] [INSPIRE].

[9] C. Herdeiro, E. Radu and H. Runarsson, Non-linear Q-clouds around Kerr black holes, Phys. Lett. B 739 (2014) 302 [arXiv: 1409.2877] [INSPIRE]. 
[10] P.V.P. Cunha, C.A.R. Herdeiro, E. Radu and H.F. Runarsson, Shadows of Kerr black holes with scalar hair, Phys. Rev. Lett. 115 (2015) 211102 [arXiv:1509.00021] [INSPIRE].

[11] Y. Brihaye, C. Herdeiro and E. Radu, Inside black holes with synchronized hair, Phys. Lett. B 760 (2016) 279 [arXiv: 1605.08901] [InSPIRE].

[12] J.D. Bekenstein, Black hole hair: 25-years after, in Physics, proceedings of the 2nd International A.D. Sakharov Conference, Moscow, Russia, 20-24 May 1996, pp. 216-219, (1996), gr-qc/9605059 [INSPIRE].

[13] C.A.R. Herdeiro and E. Radu, Asymptotically flat black holes with scalar hair: a review, Int. J. Mod. Phys. D 24 (2015) 1542014 [arXiv: 1504.08209] [INSPIRE].

[14] S. Hod, No-scalar-hair theorem for spherically symmetric reflecting stars, Phys. Rev. D 94 (2016) 104073 [arXiv: 1612.04823] [INSPIRE].

[15] S. Hod, No nonminimally coupled massless scalar hair for spherically symmetric neutral reflecting stars, Phys. Rev. D 96 (2017) 024019 [arXiv: 1709.01933] [INSPIRE].

[16] S. Bhattacharjee and S. Sarkar, No-hair theorems for a static and stationary reflecting star, Phys. Rev. D 95 (2017) 084027 [arXiv: 1704.02873] [INSPIRE].

[17] S. Hod, Charged massive scalar field configurations supported by a spherically symmetric charged reflecting shell, Phys. Lett. B 763 (2016) 275 [arXiv:1703.05333] [INSPIRE].

[18] S. Hod, Marginally bound resonances of charged massive scalar fields in the background of a charged reflecting shell, Phys. Lett. B 768 (2017) 97 [arXiv: 1806.06831] [INSPIRE].

[19] Y. Peng, B. Wang and Y. Liu, Studies of scalar field configurations supported by reflecting shells in the AdS spacetime, Eur. Phys. J. C 78 (2018) 680 [arXiv:1803.09148] [inSPIRE].

[20] S. Hod, Charged reflecting stars supporting charged massive scalar field configurations, Eur. Phys. J. C 78 (2018) 173 [arXiv:1801.02801] [InSPIRE].

[21] S. Hod, Stationary bound-state scalar configurations supported by rapidly-spinning exotic compact objects, Phys. Lett. B $\mathbf{7 7 0}$ (2017) 186 [arXiv: 1803. 07093] [INSPIRE].

[22] S. Hod, Ultra-spinning exotic compact objects supporting static massless scalar field configurations, Phys. Lett. B 774 (2017) 582 [arXiv:1708.09399] [INSPIRE].

[23] S. Hod, Onset of superradiant instabilities in rotating spacetimes of exotic compact objects, JHEP 06 (2017) 132 [arXiv:1704.05856] [INSPIRE].

[24] Y. Peng, Scalar field configurations supported by charged compact reflecting stars in a curved spacetime, Phys. Lett. B 780 (2018) 144 [arXiv:1801.02495] [InSPIRE].

[25] Y. Peng, Scalar condensation behaviors around regular Neumann reflecting stars, Nucl. Phys. B 934 (2018) 459 [arXiv:1805.05706] [INSPIRE].

[26] Y. Peng, Static scalar field condensation in regular asymptotically AdS reflecting star backgrounds, Phys. Lett. B 782 (2018) 717 [arXiv:1804.10787] [INSPIRE].

[27] Y. Peng, Hair formation in the noncommutative reflecting star background, arXiv: 1809.05329 [INSPIRE].

[28] S. Chandrasekhar, The Mathematical Theory of Black Holes, Oxford University Press, New York (1983).

[29] S.L. Shapiro and S.A. Teukolsky, Black Holes, White Dwarfs and Neutron Stars: The Physics of Compact Objects, first edition, Wiley-Interscience (1983). 
[30] S. Hod, Extremal Kerr-Newman black holes with extremely short charged scalar hair, Phys. Lett. B 751 (2015) 177 [arXiv: 1707.06246] [INSPIRE].

[31] V. Cardoso, L.C.B. Crispino, C.F.B. Macedo, H. Okawa and P. Pani, Light rings as observational evidence for event horizons: long-lived modes, ergoregions and nonlinear instabilities of ultracompact objects, Phys. Rev. D 90 (2014) 044069 [arXiv:1406.5510] [INSPIRE].

[32] P. Grandclément, Light rings and light points of boson stars, Phys. Rev. D 95 (2017) 084011 [arXiv: 1612.07507] [INSPIRE].

[33] J. Keir, Slowly decaying waves on spherically symmetric spacetimes and ultracompact neutron stars, Class. Quant. Grav. 33 (2016) 135009 [arXiv:1404.7036] [INSPIRE].

[34] V. Cardoso, A.S. Miranda, E. Berti, H. Witek and V.T. Zanchin, Geodesic stability, Lyapunov exponents and quasinormal modes, Phys. Rev. D 79 (2009) 064016 [arXiv:0812.1806] [INSPIRE].

[35] S. Hod, Upper bound on the radii of black-hole photonspheres, Phys. Lett. B 727 (2013) 345 [arXiv: 1701.06587] [INSPIRE].

[36] P.V.P. Cunha, E. Berti and C.A.R. Herdeiro, Light-Ring Stability for Ultracompact Objects, Phys. Rev. Lett. 119 (2017) 251102 [arXiv:1708.04211] [INSPIRE].

[37] S. Hod, On the number of light rings in curved spacetimes of ultra-compact objects, Phys. Lett. B 776 (2018) 1 [arXiv:1710.00836] [INSPIRE].

[38] S. Hod, Upper bound on the gravitational masses of stable spatially regular charged compact objects, Phys. Rev. D 98 (2018) 064014 [INSPIRE].

[39] D. Núñez, H. Quevedo and D. Sudarsky, Black holes have no short hair, Phys. Rev. Lett. 76 (1996) 571 [gr-qc/9601020] [INSPIRE].

[40] S. Hod, Hairy Black Holes and Null Circular Geodesics, Phys. Rev. D 84 (2011) 124030 [arXiv:1112.3286] [INSPIRE].

[41] A.E. Mayo and J.D. Bekenstein, No hair for spherical black holes: Charged and nonminimally coupled scalar field with selfinteraction, Phys. Rev. D 54 (1996) 5059 [gr-qc/9602057] [INSPIRE].

[42] H. Bondi, Anisotropic spheres in general relativity, Mon. Not. Roy. Astron. Soc. 259 (1992) 365.

[43] P. Basu, C. Krishnan and P.N.B. Subramanian, Hairy Black Holes in a Box, JHEP 11 (2016) 041 [arXiv : 1609.01208] [INSPIRE].

[44] N. Sanchis-Gual, J.C. Degollado, P.J. Montero, J.A. Font and C. Herdeiro, Explosion and Final State of an Unstable Reissner-Nordström Black Hole, Phys. Rev. Lett. 116 (2016) 141101 [arXiv: 1512.05358] [INSPIRE].

[45] S.R. Dolan, S. Ponglertsakul and E. Winstanley, Stability of black holes in Einstein-charged scalar field theory in a cavity, Phys. Rev. D 92 (2015) 124047 [arXiv:1507.02156] [INSPIRE].

[46] Y. Peng, Studies of a general flat space/boson star transition model in a box through a language similar to holographic superconductors, JHEP 07 (2017) 042 [arXiv: 1705.08694] [INSPIRE].

[47] Y. Peng, B. Wang and Y. Liu, On the thermodynamics of the black hole and hairy black hole transitions in the asymptotically flat spacetime with a box, Eur. Phys. J. C 78 (2018) 176 [arXiv: 1708.01411] [INSPIRE]. 